\title{
Nanotribology on Polymer Blend Surface by Atomic Force Microscopy
}

\author{
Motonori Komura, ${ }^{1, \dagger}$ Zhaobin QIU, ${ }^{2}$ Takayuki IKEHARA, ${ }^{2}$ \\ Ken NAKAJIMA, ${ }^{1}$ and Toshio Nishi ${ }^{1}$ \\ ${ }^{1}$ Department of Organic and Polymeric Materials, Graduate School of Science and Engineering, \\ Tokyo Institute of Technology, O-okayama, Meguro-ku, Tokyo 152-8552, Japan \\ ${ }^{2}$ Department of Applied Chemistry, Faculty of Engineering, Kanagawa University, \\ Rokkakubashi, Kanagawa, Yokohama 221-8686, Japan
}

(Received March 18, 2005; Accepted August 23, 2005; Published January 15, 2006)

\begin{abstract}
Nanotribological properties of polystyrene/poly(vinyl methyl ether) blend surface mainly at its melt state were investigated using frictional loop (FL) measurement by atomic force microscopy (AFM). The energy dissipation estimated from the area inside the FL showed each peak against both temperature and scan rate. Indentation depth profile against scan rate was simultaneously detected. The indentation was largely restricted above the scan rate where the dissipation energy curve had a peak. We could succeed in drawing both master curves for the energy dissipation and the indentation depth using the same shift factor $a_{\mathrm{T}}$. It was concluded that AFM is a powerful tool for evaluating the nanotribological properties of polymer melt. [DOI 10.1295/polymj.38.31]

KEY WORDS Nanotribology / Atomic Force Microscopy / Polymer Blend / Energy Dissipation / Indentation / Master Curve /
\end{abstract}

Polymers are soft-materials and have inevitably different physical properties from metals, semiconductors, ceramics and so on. Some technical terms such as viscoelasticity, elastic and plastic deformation, adhesion, friction and lubrication are therefore quite essential in polymeric materials research. Higherorder structures of polymers have characteristic nanometer-scale sizes. This is because polymers are "giant molecules" that cannot be fully understood merely in terms of atoms or low-molecular-weight molecules. Thus, to promote "polymer nanotechnology," chanical property studies such as rheology and tribology of polymers are of great importance. Research in these fields has been reported using macroscopic instruments such as dynamic mechanical analyzer (DMA), surface force apparatus (SFA) and ball indentation method to date.

When friction occurs between two surfaces in a macroscopic scale, both surfaces contact at numerous asperities in most cases. Therefore, apparent and realistic contact areas are different from each other due to their macroscopic roughness. To understand this phenomenon, investigation of tribological properties at a single asperity contact is very important. Scanning probe microscopy (SPM) allows one to systematically investigate surface and interfacial properties on atomic and nanoscopic scales. Atomic force microscopy $(\mathrm{AFM})^{2}$ and frictional force microscopy $(\mathrm{FFM})^{3}$ are designed for measuring the force between a probe tip and a specimen surface both statically and dynam- ically as well as the topographic pattern of surfaces.

We have been investigating AFM and FFM as powerful tools for evaluating nanomechanical properties of polymeric materials, especially in their melt states, namely, at temperatures above their glass-transition temperatures, $T_{\mathrm{g}}$. Basically, both methods are used to obtain "microscopic images" of surfaces and applied to hard (or non-soft) materials. Our attention has been directed to mechanical properties of soft materials, such as viscoelasticity, adhesive force, friction, surface deformation, etc. In the Kajiyama group, many studies on mechanical properties of polymeric materials measured by AFM have been reported. ${ }^{4-9}$ Lower $T_{\mathrm{g}}$ on a surface of polymeric materials than the bulk $T_{\mathrm{g}}$ has been finely demonstrated through lateral force measurements by AFM. ${ }^{5}$ These studies have been conducted in the glass state of polymeric materials below the bulk $T_{\mathrm{g}}$. Previous articles ${ }^{10,11}$ measured forcedistance curves (FCs) and frictional loops (FLs) of polystyrene/poly(vinyl methyl ether) (PS/PVME) blends in glass, glass-rubber transition and rubber (melt) states by AFM and FFM. The present research, reports the nanotribological properties of PS/PVME blends in more detail including temperature and scan rate dependence of FLs. We will discuss that change in intimateness of contact caused by a tip indentation into a polymeric surface remarkably contributes to the friction of polymer melt.

${ }^{\dagger}$ To whom correspondence should be addressed (E-mail: komura-pd@pfc.res.titech.ac.jp). 


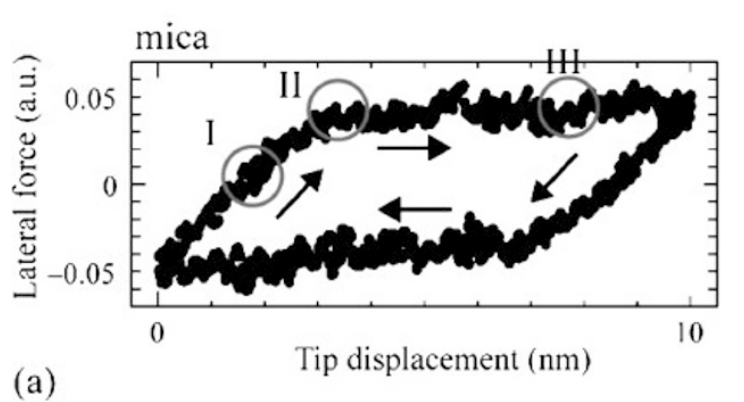

(a)

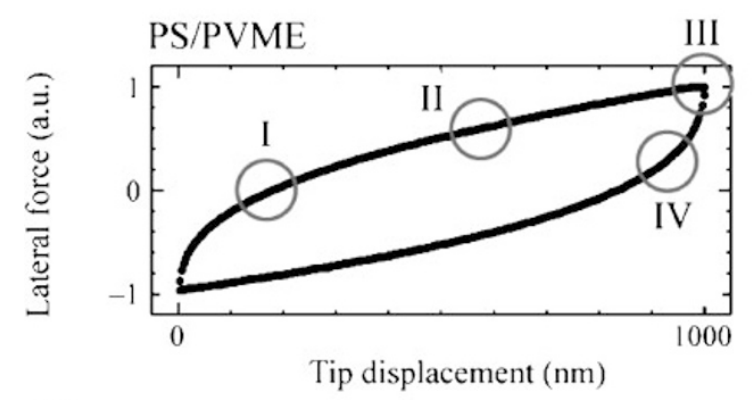

(b)

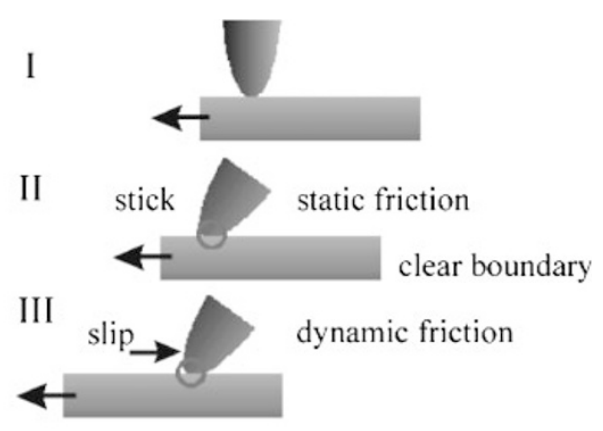

I

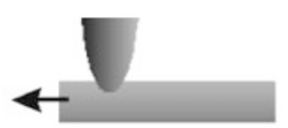

II

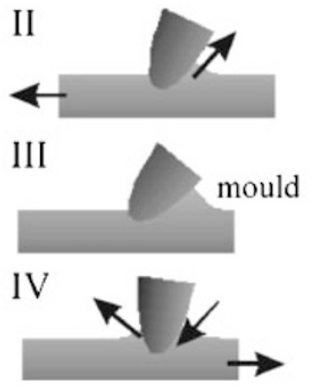

Figure 1. Frictional loops for (a) mica surface and (b) PS/PVME blend surface. For the former, a clear boundary between static and kinetic frictions was observed, while it disappeared in the latter due to large deformation.

\section{EXPERIMENTAL}

PS/PVME blends were prepared with PS (TOSOH Corp., Japan) with $M_{\mathrm{W}}=190,000$ and PVME (Polysciences Inc., PA, USA) with $M_{\mathrm{W}}=99,000$. The degrees of polymerization of both samples were almost same. $T_{\mathrm{g}} \mathrm{s}$ of PS and PVME were about 100 and $-24{ }^{\circ} \mathrm{C}$, respectively. $T_{\mathrm{g}}$ determines the mechanical properties of polymers: polymers below $T_{\mathrm{g}}$ are hard glassy materials with GPa-order elastic modulus, while they become soft rubbery with MPa-order elastic modulus above $T_{\mathrm{g}}$. Since this blend had a phase diagram of lower critical solution temperature (LCST) and was miscible below $120^{\circ} \mathrm{C}, T_{\mathrm{g}}$ and its viscoelastic properties could be widely changed with its blend ratio. Therefore, it is an appropriate model sample for measurement of mechanical properties of polymeric materials. Since our AFM instrument controls an environmental temperature from room to higher (about $200^{\circ} \mathrm{C}$ ) temperature, we mainly report the results of the sample with the PS content of 60\% (PS60) whose $T_{\mathrm{g}}$ was $47^{\circ} \mathrm{C}$ measured by differential scanning calorimetry (DSC2910, TA instruments, DE, USA). The blend samples were prepared using toluene as a mutual solvent. The solution was cast onto freshly cleaved mica substrate and spin-coated at $3000 \mathrm{rpm}$ for $1 \mathrm{~min}$. Each film was dried for one day at $80^{\circ} \mathrm{C}$ in a vacuum chamber.
All AFM data were obtained by contact-mode operation with NanoScope IV (Veeco Instruments, CA, USA). We used cantilevers with a pyramidal $\mathrm{Si}_{3} \mathrm{~N}_{4}$ tip and a normal spring constant of $0.1 \mathrm{~N} / \mathrm{m}$ (OMCLRC800PS, Olympus, Japan). All measurements were performed under dried nitrogen gas to exclude the effects of vapor and oxidation.

In frictional loop (FL) measurement, after a normal load of $1.6 \mathrm{nN}$ was given to an AFM cantilever, its probe tip was scanned laterally on a polymer blend surface. We measured the lateral bend of the cantilever (torsion) as a function of lateral displacement of the sample. Lateral force was in an arbitrary unit, because the nominal value of torsional spring constant is generally unreliable and direct measurement of torsional spring constant has not been well established. Scanning length was set at $1 \mu \mathrm{m}$.

\section{RESULTS AND DISCUSSION}

FL of a mica surface as an example of hard materials is shown in Figure 1a. To know the features well, the scan range was set to $10 \mathrm{~nm}$. The shape of FL seemed like a parallelogram. As schematically shown, in the region from I to II a probe tip stuck to the surface and static friction was observed. At the point II, torsional force of a cantilever overcame the adhesive force between the tip and surface and the tip began to slip. In the region from II to III, kinetic friction 
was observed. In the case of mica, there was a clear boundary between static and kinetic frictions.

Figure $1 \mathrm{~b}$ shows a typical FL of PS60. Contrary to the results for mica, there was no clear boundary and the lateral force gradually increased on scanning. As schematically shown, when a tip slides over a soft polymer surface, a mould is formed and grows at the front rim of the tip. The growth leads to increment of the lateral force. Thus, in the case of soft materials the deformation of specimen surface contributes to friction. Classical theory introduced by Bowden and Tabor $^{12}$ describes that friction is proportional to a real contact area that is regarded as JKR contanct. ${ }^{13}$ Such a theory, however, is obviously not applicable to a largely deformed system. Though there is a theoretical framework that considers surface deformation by Ludema and Tabor, ${ }^{14}$ the idea is also based on a macroscopic way of thinking and thus not enough to be used for previous results. We should have a certain theory capable for discussing nanometer-scale deformation. One of our co-workers reported a computer simulation based on molecular dynamics and reproduced a mould formation. ${ }^{15}$

Figure 2 shows the temperature dependence of FL of PS60 at the scan rate $2.5 \mathrm{~Hz}$. At $75^{\circ} \mathrm{C}$ the area inside the FL showed a peak somewhat unexpected because all measurements were performed above $T_{\mathrm{g}}$ and thus there was no specific "phase transition." This implies that there exists some contribution to the frictional phenomenon. Although a more precise answer will be given later, we qualitatively interpret the result here. As known through conventional force-distance

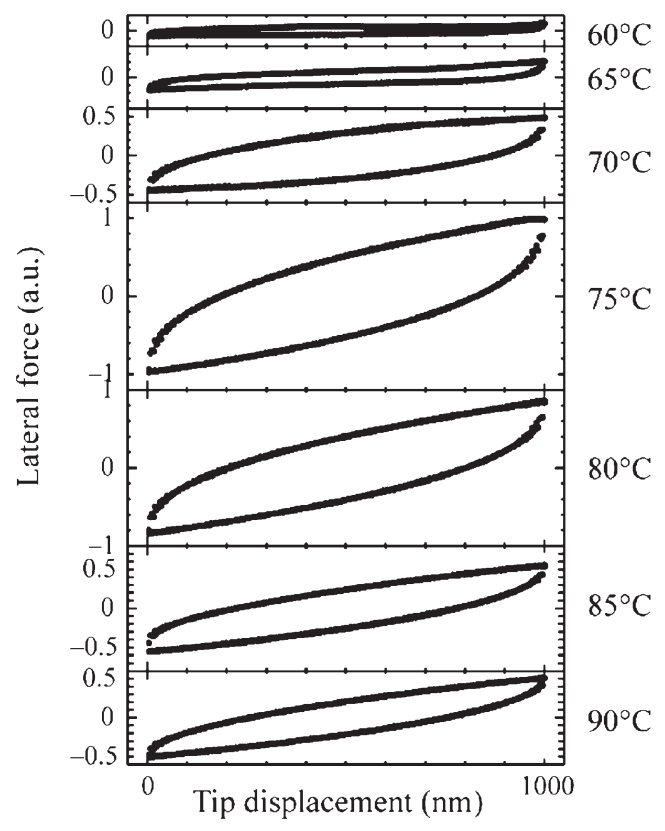

Figure 2. A series of FL with elevated temperature. Scan size and scan rate were kept constant at $1 \mu \mathrm{m}$ and $2.5 \mathrm{~Hz}$, respectively. The area inside FL shows maximum at $75^{\circ} \mathrm{C}$. curve measurements (data not shown), with elevating temperature the specimen surface becomes more easily deformed due to decreased elastic modulus. The contact area between the tip and surface and adhesive force thus become larger. Therefore, the frictional force increases. The lateral resisting force to deform the surface decreases with temperature for the same reason. Because of the competition between the two effects, frictional force meets its maximum at a certain condition. The energy dissipation of friction (frictional energy) is generally given as kinetic frictional force times scan length. Since the restoring energy accumulated in the cantilever is cancelled when an area inside a FL is calculated, the area can be evaluated as energy dissipation. In summery, the energy dissipation had a peak at $75^{\circ} \mathrm{C}$ in this measurement.

Figure 3 shows the scan rate dependence of FL of PS60 at $75^{\circ} \mathrm{C}$. The area inside FL again showed a peak at a certain scan rate around $2 \mathrm{~Hz}$. The relationship was replotted in terms of energy dissipation and is shown in Figure 4. As expected, there exists remarkable similarity between temperature dependence and scan-rate dependence. Thus, "time-temperature superposition principle," which is quite specific for polymeric materials, is valid even in nanoscopic world.

To confirm the above competition, we investigated tip indentation into a surface. An average value of height associated with z-piezo scanner during one frictional loop was defined as "indentation depth" because normal load applied for all measurements is

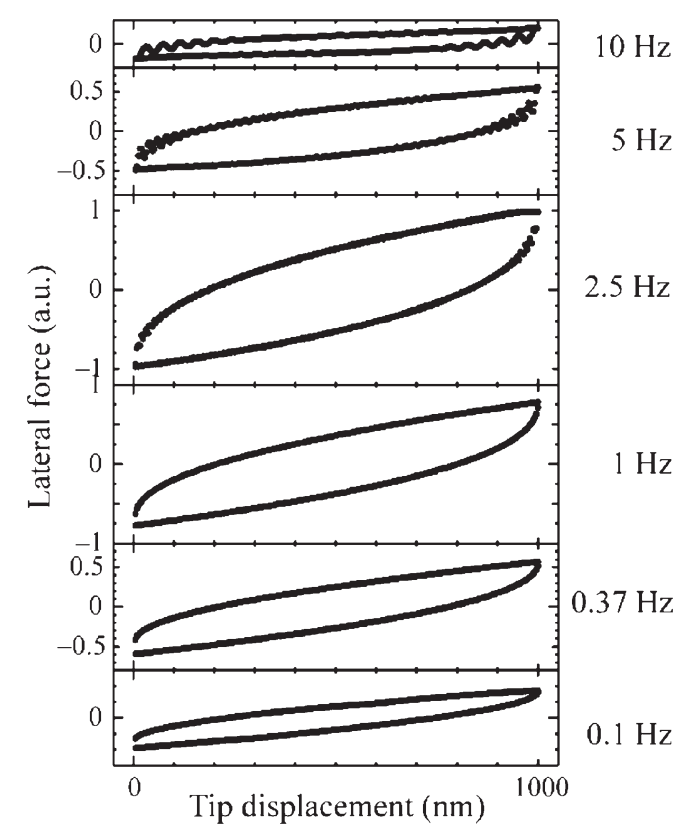

Figure 3. The dependence of FLs on scan rate. Scan size was $1 \mu \mathrm{m}$ and the temperature was kept at $75^{\circ} \mathrm{C}$. The behavior similar to Figure 2 may be due to "time-temperature superposition principle." 

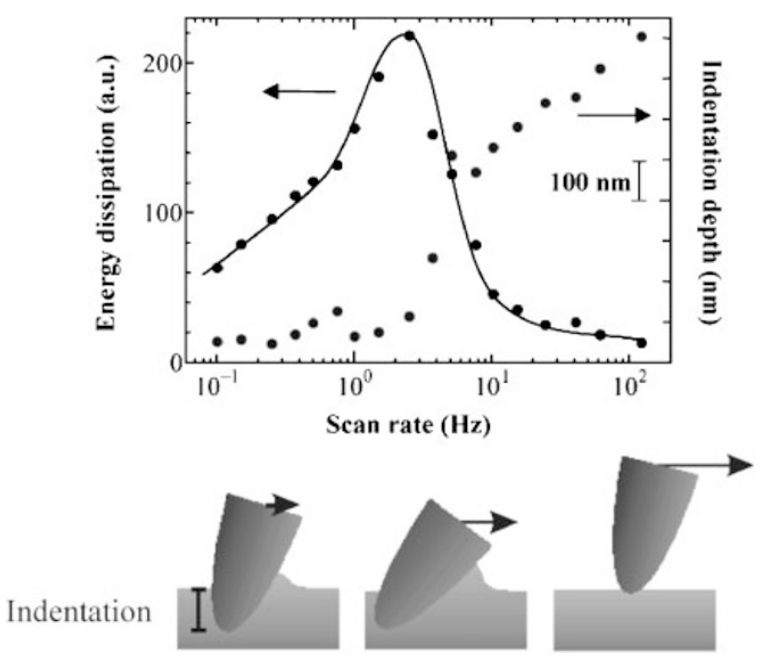

Figure 4. The relationship between the energy dissipation estimated from Figure 3 and scan rate. Measurement of indentation depth was performed and is superimposed in the same graph.

identical and thus cantilever deflection was kept constant. Scan rate dependence of the indentation depth at the sample temperature of $75^{\circ} \mathrm{C}$ is superimposed in Figure 4. Since the position of an uppermost surface could not be detected because the sample deforms by a normal load of $1.6 \mathrm{nN}$, a relative value for each scan rate was plotted. The behavior of the indentation depth profile remarkably changed at a scan rate where the energy dissipation peaked. Large indentation depth was observed below the peak and was almost constant (reason unknown; the sample thickness of $2 \mu \mathrm{m}$ and probe tip height of about $4 \mu \mathrm{m}$ are sufficiently larger than this value). Therefore, the contact area between the tip and surface was also constant. Consequently, increment in energy dissipation with increasing scan rate (left side of the peak) was due to that of resisting force mainly caused by effective elastic mod- ulus change. The indentation was suppressed at much higher scan rates (right side of the peak) due to further change in elastic modulus, which caused decrement in frictional force due to smaller contact area and thus smaller adhesion.

The reason for change in indentation depth is probably stick-slip of a probe tip on a specimen surface. Torsional force of a cantilever gradually increases on scanning, because the mound of the sample grows. When the force overcomes the adhesive force between the tip and surface, the tip is detached from the surface and has a new contact with the next position of the surface along the scan direction. Since the temporal detachment causes decrement of the contact area, the cooperative effect makes contact more easily broken. Since the cantilever more largely twists at higher scan rates even on the same scanning length because of larger elastic modulus, the slip more easily occurs. Therefore, indentation is restricted at higher scan rates. To our knowledge, this is the first report that friction of a polymeric material was discussed on the basis of depth information of samples evaluated by AFM. In nanometer scale, tribological properties are strongly correlated with sample deformation, effective elastic modulus and adhesion, namely, rheological parameters.

Temperature dependence of the frictional energy curve and indentation depth profile are shown in Figure 5. Both curves shifted to higher scan rates with increasing temperature. Since the surface is more easily deformed with increasing temperature, contact between the tip and surface becomes closer so that slip hardly occurs. Therefore, large indentation takes place even on higher scan rates and both curves shift to the right. Because a sample stage had to be separated from a tip using a stepping motor equipped with a
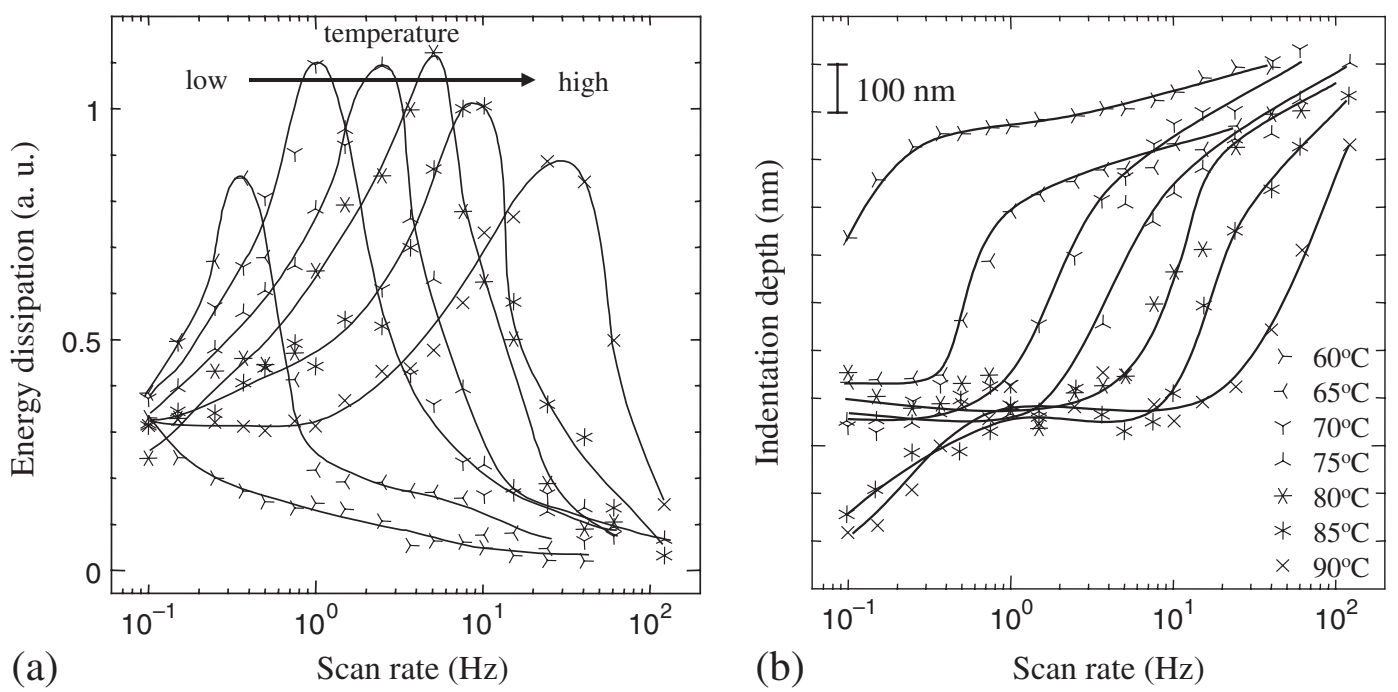

Figure 5. (a) Dependence of energy dissipation curves on temperature. (b) That of indentation depth. Indentation depth was not the real depth from an uppermost surface but a relative value to each other. 

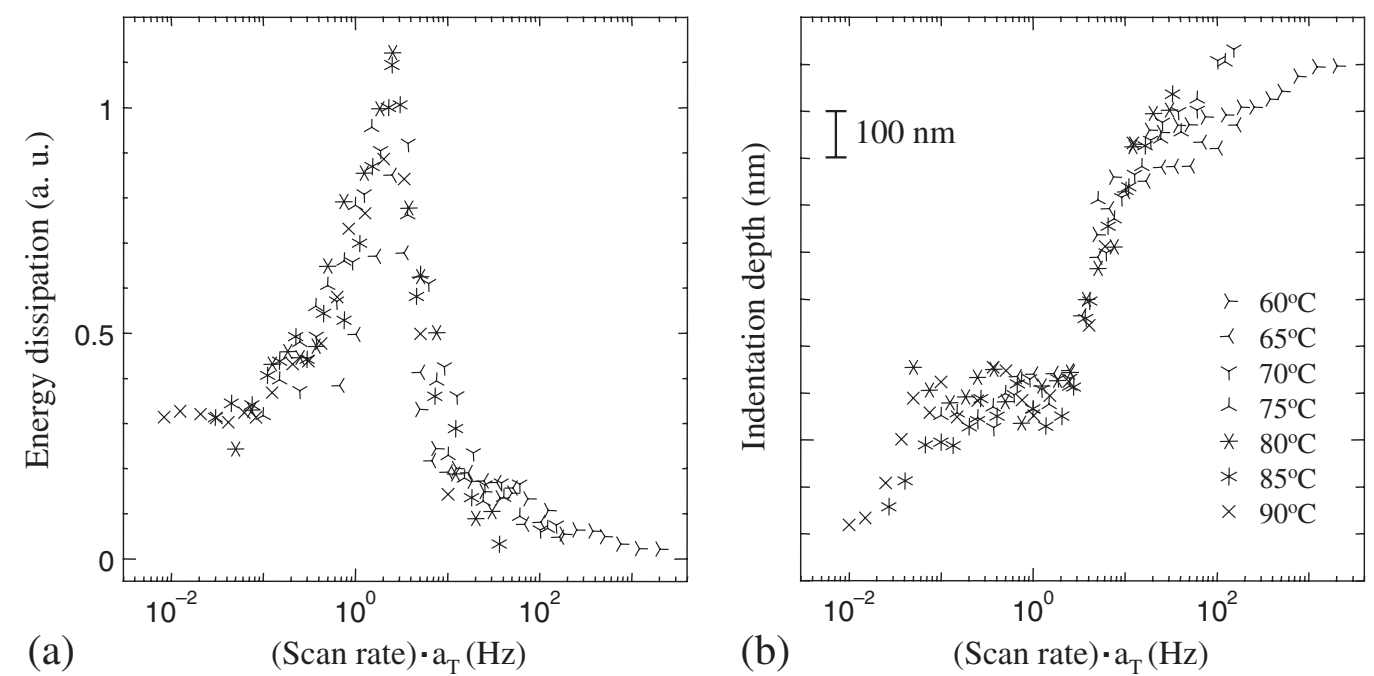

Figure 6. Master curves for (a) Figure 5a and (b) Figure 5b, respectively were drawn using the same shift factor, $a_{\mathrm{T}}$.

z-piezo scanner when the sample temperature was changed, absolute values of indentation depth at different temperatures could not be directly compared. Therefore, vertical shifts for each curve were meaningless and shifted along the vertical axis merely for eye guide.

Figure 6a shows the master curve for energy dissipation curves determined by shifting each curve to the curve at reference temperature, $75^{\circ} \mathrm{C}$. Since a good master curve was obtained, we were quite sure that "the time-temperature superposition principle" could be applied to the friction of polymer melt in nanometer scale. A series of the same shift factor $a_{\mathrm{T}}$ was applied to the indentation depth curves as shown in Figure 6b. Two different master curves were drawn by the same $a_{\mathrm{T}}$. Both physical parameters must have the same origin.

In macroscopic systems, the origin of frictional force peak against velocity or temperature is thought to be due to $\alpha$-relaxation of polymer chains. ${ }^{16}$ Figure 7 shows the result fitting to the WLF equation, ${ }^{17}$

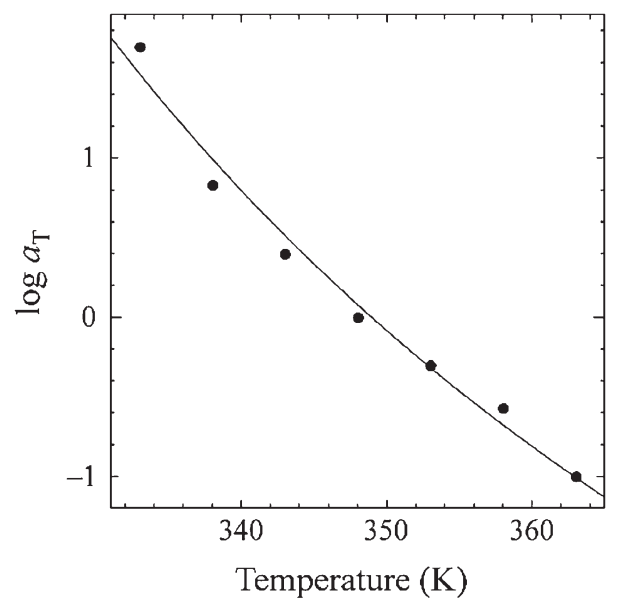

Figure 7. Curve fitting of shift factor, $a_{\mathrm{T}}$ to WLF equation.

$$
\log a_{\mathrm{T}}=-\log a_{\mathrm{T} 0}-\frac{8.86 \times\left(T-T_{\mathrm{S}}\right)}{101.5+T-T_{\mathrm{S}}}
$$

where $T_{\mathrm{s}}=T_{\mathrm{g}}+50^{\circ} \mathrm{C}$ and $a_{\mathrm{T} 0}$ is a shift factor of the curve at $75^{\circ} \mathrm{C}$. $T_{\mathrm{s}}$ is the reference temperature. The fitting resulted in $T_{\mathrm{g}} \sim 15^{\circ} \mathrm{C}$, which is lower than that obtained by DSC, $47^{\circ} \mathrm{C}$. Though the difference could be explained due to the segregation of PVME moiety to the surface and/or the increment of molecular mobility at the surface owing to more free space, these phenomena were effective in the region of a few $\mathrm{nm}$ from an uppermost surface. Since our experiment was conducted in much deeper region, the lower $T_{\mathrm{g}}$ might be explained by different reasons. Application of the WLF equation might not be adequate for the friction of polymer melt in nanometer scale.

One may notice the sharpness of the master curve in Figure 6a compared with that by conventional macroscopic measurement. ${ }^{16}$ We speculate that the friction measurement by AFM probe tip ideally corresponds to a single asperity measurement. To elucidate this point, the dependence of energy dissipation curve on applied load was investigated (data not shown). With increasing applied load, the curve shifted to higher scan rates, because the tip indentation is enhanced by larger applied load. If macroscopic friction is realized by multiple asperities and there is distribution of applied load at each asperity, the width of master curve must be broadened. To mediate macroscopic and nanoscopic friction phenomena, however, both experimental and theoretical studies must be conducted more extensively.

\section{CONCLUSIONS}

We investigated temperature and scan rate dependence of the frictional loop for PS/PVME blend sur- 
face by AFM. Energy dissipation curves against temperature and scan rate had each peak. AFM tip indentation was largely suppressed above the scan rate where the frictional energy curve had a peak. We succeeded in clarifying contribution of surface deformation to the friction of a polymer melt. Tribology of polymeric materials in nanometer scale must be discussed with rheological consideration. We conclude that AFM is a powerful tool for evaluating the nanomechanical properties of a polymer melt.

Acknowledgment. The work was supported by National Institute of Advanced Industrial Science and Technology (AIST), Japan Chemical Innovation Institute (JCII) and New Energy Development Organization (NEDO) as one of the projects in the Nanotechnology Program by the Ministry of Economy, Trade, and Industry (METI) of Japan.

\section{REFERENCES}

1. T. Nishi, Nippon Secchaku Gakkaishi, 38, 193 (2002).

2. G. Binnig, C. F. Quate, Ch. Gerber, and E. Weibel, Phys. Rev. Lett., 56, 930 (1986).

3. C. M. Mate, G. M. McClelland, R. Erlandsson, and S. Chiang, Phys. Rev. Lett., 59, 1942 (1987).
4. K. Tanaka, A. Takahara, and T. Kajiyama, Macromolecules, 30, 6626 (1997).

5. K. Tanaka, A. Takahara, and T. Kajiyama, Macromolecules, 33, 7588 (2000).

6. N. Satomi, K. Tanaka, A. Takahara, T. Kajiyama, T. Ishizone, and S. Nakahama, Macromolecules, 34, 8761 (2001).

7. K. Tanaka, S. Dai, T. Kajiyama, K. Aoi, and M. Okada, Langmuir, 19, 1196 (2003).

8. T. Kajiyama, K. Tanaka, and A. Takahara, J. Polym. Sci., Part A: Polym. Chem., 42, 639 (2004).

9. A. Sakai, K. Tanaka, Y. Fujii, T. Nagamura, and T. Kajiyama, Polymer, 46, 429 (2005).

10. K. Nakajima, H. Yamaguchi, J. C. Lee, M. Kageshima, T. Ikehara, and T. Nishi, Jpn. J. Appl. Phys., 36, 3851 (1997).

11. Y. Terada, M. Harada, T. Ikehara, and T. Nishi, J. Appl. Phys., 87, 2803 (2000).

12. F. P. Bowden and D. Tabor, "The Friction and Lubrication of Solids," Pt. 1 Oxford University, New York, 1950.

13. K. L. Johnson, K. Kendall, and A. D. Roberts, Proc. R. Soc. London, A324, 301 (1971).

14. K. C. Ludema and D. Tabor, Wear, 9, 329 (1966).

15. M. Morita, T. Ikehara, T. Nishi, and M. Doi, Polym. J., 36, 265 (2004).

16. K. A. Grosch, Proc. R. Soc. London, A274, 21 (1963).

17. M. L. Williams, R. F. Landel, and J. D. Ferry, J. Am. Chem. Soc., 77, 3701 (1955). 